\title{
Quantification of Energy Cost Savings through Optimization and Control of Appliances within Smart Neighborhood Homes
}

\author{
Supriya Chinthavali*, Varisara Tansakul*, Sangkeun Lee*, Anika Tabassum ${ }^{\square}$, Jeff \\ Munk* $^{*}$ Jan Jakowski ${ }^{\triangle}$, Michael Starke*, Teja Kuruganti*, Heather Buckberry* ${ }^{*}$ Jim \\ Leverette ${ }^{\dagger}$ \\ *Oak Ridge National Laboratory, $\quad{ }^{\Delta}$ University of Tennessee, Knoxville, $\quad{ }^{\dagger}$ Southern Company, $\quad{ }^{\square}$ Virginia \\ Tech \\ Email: \{chinthavalis, tansakulv, lees4, munkjd, starkemr, kurugantipv, buckberryhl\}@ornl.gov, \\ anikat1@vt.edu, jasjakowski@gmail.com, jalevere@southernco.com
}

\begin{abstract}
Electric utilities are driving towards enabling automatic scheduling and control of the consumption pattern of appliances such as heating, ventilation, and air conditioning (HVAC) and water heater (WH) systems (e.g., through preheating and pre-cooling, etc.) within smart neighborhoods to minimize energy cost and peak load demand. Quantifying economic savings through direct comparison of the optimized energy usage profile on a specific day with the typical non-optimized usage profile on another day is not a fair comparison because energy usage highly depends on weather conditions and human behaviour especially appliance like HVAC on those days. In this paper, we propose a novel approach of identifying similar weather day pairs which can then be used to compare the energy use profiles within homes between the identified pairs. We then demonstrate how the proposed approach can be used to compute cost savings due to optimization and control of smart appliances at home and neighborhood-level within a futurefocused smart neighborhood of 62 residential homes. We also demonstrate a simulation based approach to quantify cost savings and showcase our findings through customized and interactive visualizations.
\end{abstract}

\section{KEYWORDS}

Internet of Things, Smart neighborhood, sensors, HVAC, water heater, furnace

ACM acknowledges that this contribution was authored or co-authored by an employee, contractor, or affiliate of the United States government. As such, the United States government retains a nonexclusive, royalty-free right to publish or reproduce this article, or to allow others to do so, for government purposes only.

UrbSys'19, November 13-14, 2019, New York, NY, USA

(c) 2019 Association for Computing Machinery.

ACM ISBN 978-1-4503-7014-1/19/11 ..\$15.00

https://doi.org/10.1145/3363459.3363535

\section{ACM Reference Format:}

Supriya Chinthavali*, Varisara Tansakul*, Sangkeun Lee*, Anika Tabassum ${ }^{\square}$, Jeff Munk ${ }^{*}$, Jan Jakowski ${ }^{\triangle}$, Michael Starke*, Teja Kuruganti*, Heather Buckberry* ${ }^{*}$ Jim Leverette ${ }^{\dagger}$. 2019. Quantification of Energy Cost Savings through Optimization and Control of Appliances within Smart Neighborhood Homes. In 1st ACM International Workshop on Urban Building Energy Sensing, Controls, Big Data Analysis, and Visualization (UrbSys'19), November 13-14, 2019, New York, NY, USA. ACM, New York, NY, USA, 10 pages. https: //doi.org/10.1145/3363459.3363535

\section{INTRODUCTION}

The recent development of Internet of Things (IoT) technologies and big data solutions combined with the rapid modernization of the electric grid, (such as the integration of distributed energy resources (DER)), provides an unprecedented opportunity to optimize energy usage. The combination of these technologies within the scope of smart homes provides a channel for innovation in energy optimization tailored to residential energy usage patterns.

Smart sensors and appliances enable real-time data collection which can be used in optimizing and predicting energy consumption. HVAC accounts for a large portion of a home's electricity consumption (32\% in US homes [5]) and also contributes to summer and winter peak power usage during extreme weather periods. Peak power periods often require the use of expensive peaker plants that are only used for a

\footnotetext{
This manuscript has been authored by UT-Battelle, LLC under Contract No. DE-AC05-00OR22725 with the U.S. Department of Energy. The United States Government retains and the publisher, by accepting the article for publication, acknowledges that the United States Government retains a nonexclusive, paid-up, irrevocable, world-wide license to publish or reproduce the published form of this manuscript, or allow others to do so, for United States Government purposes. The Department of Energy will provide public access to these results of federally sponsored research in accordance with the DOE Public Access Plan (http://energy.gov/downloads/doe-public-accessplan).
} 
handful of days per year [6]. This results in electric utility generation costs to be exponentially higher during these periods. To help offset these costs and discourage electricity use during typical peak hours, electric utilities offer Time of Use (TOU) pricing to customers. TOU pricing is typically cheaper than the standard rate during off-peak hours and more expensive during peak demand times (typically between 10 a.m. to 7 p.m) [3]. A lot of literature focuses on optimizing smart devices such as HVAC within IoT space [12, 15].

In 2017, Southern Company, a major public electric utility, in collaboration with the US Department of Energy (DOE) and Oak Ridge National Laboratory (ORNL), planned the development and demonstration of a neighborhood-level transactive energy and controls research platform to explore grid integration, distributed control and end-use energy management [10]. This Smart Neighborhood ${ }^{\mathrm{TM}}$ of 62 homes is currently powered by both the traditional electric grid as well as a co-located microgrid, which is composed of solar panels, battery storage and a natural gas generator. This microgrid is capable of powering the entire community if necessary [4]. The goal is to co-optimize energy cost, comfort, environment, and reliability by controlling grid generation assets and homeowner end devices through transactive control. By the end of 2018, the neighborhood was operational with active dispatch and control of smart appliances, such as intelligent HVAC and heat pump water heater systems. The neighborhood is being operated with optimization dispatch enabled for a week followed by a week with optimization dispatch disabled (baseline) to collect sufficient baseline and optimization data for comparison.

The approach for optimization at the Smart Neighborhood includes a house-level and microgrid optimization. The microgrid provides a 24-hour electricity price forecast to all of the homes. The house-level optimization then uses that price forecast along with the weather forecast, building and equipment models to optimize their usage to minimize costs. The homeowner's comfort constraints are read directly from the heating and cooling set points stored in the schedule programmed into their thermostats. A mixing valve on the outlet of the $\mathrm{WH}$ allows the optimization to store additional energy in the water heating by heating to a higher temperature, while ensuring the hot water delivered to the house is not too hot. The power forecasts from all of the house-level optimization are aggregated and returned to the microgrid controller. If the load-shape is not desirable, the microgrid adjusts its 24-hour price forecast and sends it back to homes. This iterative process continues until the desired load shape is achieved or the load shape no longer changes significantly to changes in price (meaning occupant comfort limits will not allow any further load shifting). Additional details on the optimization, agent-based framework, and communications can be found in [14]. This optimization dispatch process is expected to yield significant cost savings for the utility as well as customers which we aim to quantify systematically and study the pros and cons of optimization.

In this paper, we propose to address the problem of quantifying cost savings due to the automatic control and optimization of WH and HVAC energy usage through novel approaches. We apply several statistical techniques and simulation based model to detect and quantify the cost savings when optimization is applied to the smart neighborhood by controlling their HVAC and WH settings. For the similar weather identification model that is based on statistics, we perform a direct comparison of the energy usage between pairs of similar weather days/weeks determined. For the simulation based model, we simulate the energy usage by computing how much energy would be typically required to keep the user within a defined comfortable temperature range for HVAC and WH system without optimization and control and comparing this with the Home Climate System (HCS) model when it is enabled and the HVAC and WH systems are optimized and controlled. Our initial findings indicate that significant cost savings can be obtained through HVAC and WH optimization and control by using these models for more accurate quantification of cost savings.

The remainder of this paper is organized as follows. In Section 2, we discuss the two approaches taken to quantify cost savings through optimization and control of HVAC and $\mathrm{WH}$ systems. This will include the statistical techniques to identify similar weather day/week pairs using several weather attributes as well as simulation based techniques. In Section 3, we discuss the HVAC cost savings obtained after application of these approaches within the Alabama connected neighborhood. In Section 4, we present the three bucket visualization dashboard to demonstrate the comfort, cost and the peak demand impacts at a home-level using approaches described in the previous section. Section 6 summarizes our contributions and discusses future work.

\section{METHODS}

In order to quantify the energy cost savings of our optimization, a baseline is needed to ensure that the comparison is reasonable. We propose two different approaches to establish baselines for comparison of HCS effectiveness.

Since energy demand highly depends on weather conditions $[8,13]$, we develop a methodology to calculate the similarity of two dates by leveraging various sensor data (e.g., ambient temperature, global horizontal irradiance (GHI), relative humidity, wind speed, rainfall, etc.) collected from a weather station located at the microgrid near the neighborhood. Details will be discussed in Section 2.1. 


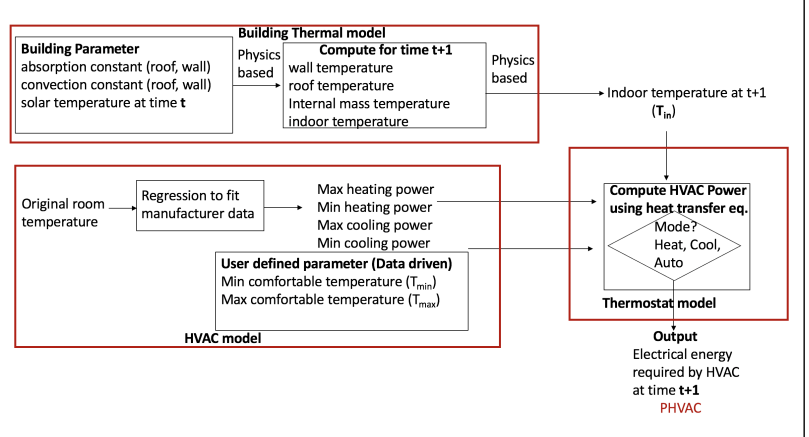

(a) HVAC simulation model
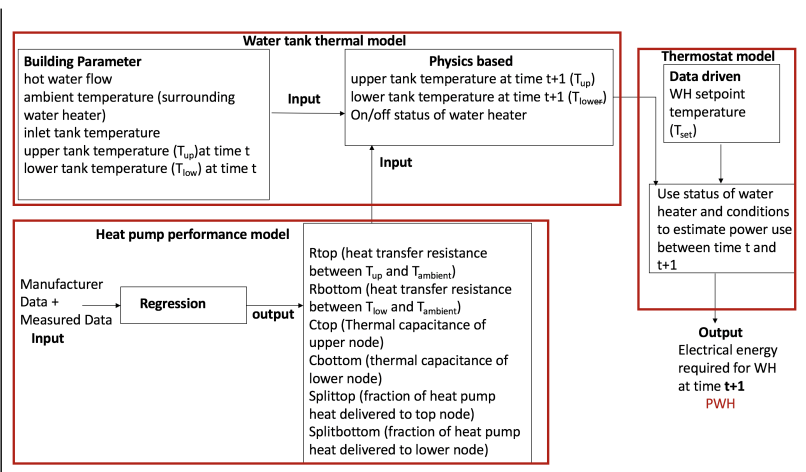

(b) WH simulation model

Figure 1: Flow chart of baseline simulation model showing how we calculate electrical energy/power for (a) HVAC (b) WH

In addition, we develop a simulation based baseline model that can be used for the comparison. The inputs for the simulation model (such as weather forecast, comfort constraints, building parameters, etc.) are same as the those used for the optimization model deployed within smart homes, but is intended to estimate the typical operation of the equipment. This approach allows a baseline day to be created that has identical weather, comfort limits, occupancy profiles, etc. Details will be discussed in Section 2.2.

\subsection{Calculating Weather Similarity}

We propose the identification of similar weather days/weeks pairs as an approach to compare energy consumption during the pair days with and without optimized control of HVAC with the assumption that power consumption patterns for HVAC of similar weather days will be comparable. To compute the pair dates, we consider weather based parameters that directly affects the comfort of the user. Extreme weather events manifest as changing weather parameters such as temperature, humidity etc which will be used as features for the computation of paired dates.

To calculate weather similarity, weather needs to be formally defined. We define weather of a specific time period $p$ as a set of arrays $W=\left\{s_{1}, s_{2}, \ldots s_{n}\right\}$, where each array $s_{i}$ is a time-series data collected from a sensor (e.g., thermometer, humidity sensor) for the specific time period $p$ and $n$ is the number of sensors. If we assume that the data is collected every 5 minute, when $p$ is a 1 -day period, the $\left|s_{i}\right|$ will be 288 $(12 \times 24)$. If $p$ is a 1 -week period, then the $\left|s_{i}\right|$ will be 2016 $(12 \times 24 \times 7)$. For example, let us assume that we employ two sensors, temperature and humidity sensors. If we pick the 1st January of 2019, the weather on this day is represented as a set of arrays $W_{01 / 01 / 2019}=\left\{s_{\text {temperature }}, s_{\text {humidity }}\right\}$, where $\left|s_{\text {temperature }}\right|=12 \times 24$ and $\left|s_{\text {humidity }}\right|=12 \times 24$.

Now the quantification of similarity of weather of two periods (e.g., two days or two weeks) is done as follows. Let us say that weather of period $p_{i}$ is $W_{i}=\left\{s_{(i, 1)}, \ldots, s_{(i, k)}, \ldots, s_{(i, n)}\right\}$ and $p_{j}$ is $W_{j}=\left\{s_{(j, 1)}, s_{(j, 1)}, \ldots, s_{(j, k)}, \ldots s_{(j, n)}\right\}$ respectively, and $s_{i, k}$ and $s_{j, k}$ constructed from the same sensor (e.g., temperature sensor). Similarity of two given arrays $u$ and $v$, $S(u, v)$ can be quantified in many ways as follows.

- Average of Mean Absolute Error (MAE): We measure the MAE [16] for every pair of elements with the same index in $u$ and $v$ and compute the average of MAEs. The value becomes zero if all elements are identical.

- Euclidean distance (ED) is the distance between two points in Euclidean space and calculated using the equations below $\left.E D=\sum\left(\left|\left(u_{i}-v_{i}\right)\right|^{2}\right)\right)^{1 / 2}$. The value becomes zero if two points are identical.

- Correlation is used to measure the dependency between two variables or in this case time series [11]. We explored three types of correlations including Kendall [1], Pearson [2], and Spearman [7]. Correlation values generally ranges from negative one to one. Correlation value of zero indicates that the time series are not correlated at all and value of one indicates that they are perfectly correlated.

- Dynamic Time Warping (DTW) measures distance between two time series, and matches shapes while taking time shifting into account [9].

With a similarity measure $S$, we can quantify the similarity of two arrays collected from each type of sensor on different periods $p_{i}$ and $p_{j}$ by calculating $S\left(s_{(i, 1)}, s_{(j, 1)}\right), S\left(s_{(i, 2)}, s_{(j, 2)}\right)$, $\ldots, S\left(s_{(i, n)}, s_{(j, n)}\right)$.

Each method results in different range. Thus, we normalize using the minimum and maximum scaler to scale scores to the given range. The normalized similarity values ranges from zero to one, where one means identical and zero means the opposite and calculate the weighted summation to have a aggregated similarity score $\left(\Sigma w_{1}, w_{2}, \ldots, w_{n}=1\right)$. We can use the same weights for all sensors or give more weights to certain sensor data (e.g., temperature and humidity) using 
domain knowledge of experts' feedback. Note that we can use multiple similarity measures together by doing one more level of weighted summation by giving different weights to different similarity measures.

We implemented the weather similarity calculation in Python. Assigning weights to different sensors and similarity measures can affect the similarity measure; however, finding the optimal weights is out of this paper's scope. We will discuss the details of our results in 3.1.

\subsection{Simulation Model}

To compare the effectiveness of the HCS optimization, we simulate the operation of the equipment without the optimization dispatch. This baseline simulation uses the same inputs as the optimization (weather forecasts, building model parameters, comfort requirements, etc.) and the same models as the optimization, but with control algorithms that mimic that of typical operation. Since the baseline simulation uses the same inputs as the optimization, the results of the two approaches can be directly compared without concerns of differences in weather or occupant behavior. This approach eliminates the error associated with inaccuracy of weather forecasts and building and equipment models. The price forecast that was used by the optimization is applied to the baseline simulation to calculate the energy cost associated with non-optimized operation. The accuracy of the forecasts and model parameters used in both the optimization and baseline simulation will ultimately affect the cost savings that are realized in the actual homes.

The simulation model consists of two modules: computation of electrical energy needed to maintain temperature settings for the HVAC shown in Figure 1(a), and a computation of the energy needed for the WH shown in Figure 1(b). We discuss these two modules in detail.

The HVAC model is composed of three components:

- Building thermal model -this is a "grey-box" model that is used to represent the heat transfer dynamics of the building. A detailed schematic of the model can be found in [14]. The building model and parameters used in the baseline simulation are identical to those used in the optimization.

- HVAC model -this consists of polynomial regressions of manufacturer performance data for power and heating/cooling capacity of the equipment. Since the HVAC system utilizes a variable speed compressor, polynomials are used to capture the minimum and maximum power and capacity as a function of outdoor air temperature. The HVAC model used for the baseline simulation is identical to that used in the optimization.

- Thermostat model -this is used to control the HVAC system based on the indoor temperature. The cooling set point schedule used in the optimization is used as the set point for the thermostat. The thermostat controls the HVAC capacity based on how close the indoor temperature is to the set point.

The general flow of the baseline simulation is shown in Figure 1(a). At time $t$, the thermostat determines the HVAC capacity based on the current indoor temperature. This capacity is then used along with the weather forecast data at time $t$ by the building model to calculate the new indoor air temperature (in addition to other building temperatures) at time $t+1$. The HVAC model is then used to calculate the energy use of the HVAC system for the time step. The energy use is multiplied by the electricity price from the optimization for the given time.

The WH model is composed of three components similar to those of the HVAC model:

- Water tank thermal model -this is "grey-box" model that is used to represent the heat transfer dynamics of the hot water storage tank. The baseline simulation uses a two-node model to simulate the upper and lower tank temperatures separately. The optimization uses a simpler single-node model due to its linearity and ensured convergence to the global minimum during optimization. While the tank models have different structures, the overall thermal resistance and thermal capacitance of the WH models are identical.

- Heat pump performance model - this consists of linear regressions of the heat pump capacity and power use based on empirical data. Since the WH uses a heat pump, the performance of the heat pump is dependent on the ambient temperature and temperature of the water in the tank. Both the optimization and the baseline simulation use an assumed, fixed ambient temperature and the average tank temperature as the water temperature.

- Thermostat model -this consists of control logic that turns on the heat pump and the two auxiliary heating elements (upper and lower) based on the upper and lower tank temperatures and the WH set point. This control logic was derived based on empirical data.

The general flow of the baseline simulation for the WH is shown in Figure 1(b) in similar fashion to that of the HVAC. At time $t$, the thermostat model is used to calculate the operating mode of the heat pump water heater (i.e., heat pump or resistance heating) based on the current tank temperatures. The heat pump performance model is then used to calculate the heating capacity delivered to the tank, or if a resistive heating element is on, its fixed capacity is used. The water tank thermal model is then used to calculate the tank temperatures at time $t+1$ based on the heat loss from the tank, any heat added by the heat pump or resistive heating 
elements, and any heat drawn from the tank in the form of hot water delivered to the house. The energy use by the WH is then calculated using the heat pump performance model or the fixed energy use of the resistive heating element for the time step.

\section{RESULTS}
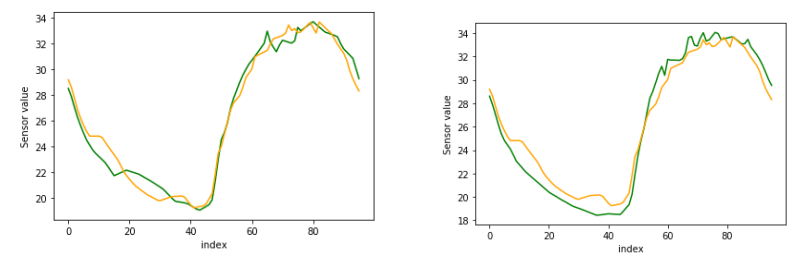

(a) Ambient Temperature May 25th (b) Ambient Temperature May 26th \& June 3rd \& June 3rd
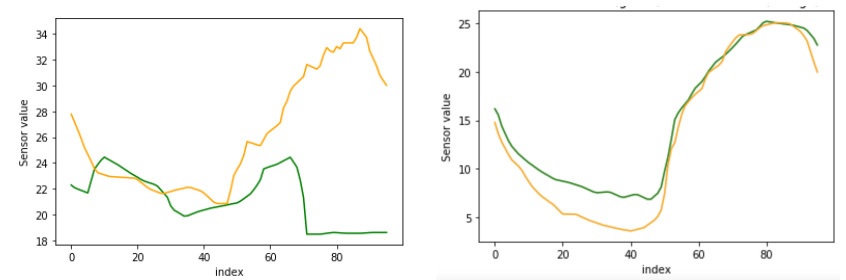

(c) Ambient Temperature May 9th \& (d) Ambient Temperature April 16th June 4th $\&$ April 21st

Figure 2: Plots of Ambient Temperature for each date pair 2(b)- 2(c), and for April 16th and April 21st (Easter Holiday) shown in 2(d). The first date of every date pair is shown in green and second date of every date pair is displayed in orange.

In this section, we analyze 62 participant homes in the same neighborhood in Alabama over a 4 month period spanning from the start of April to the end of July. We focus on energy cost comparison for HVAC since energy usage patterns of HVAC are more correlated with weather than the $\mathrm{WH}$. We present the results of our similar weather days/weeks identification software implementation in section 3.1. Then, the results of simulation models are discussed in section 3.2.

\subsection{Similar Weather Pairs (Days/Weeks)}

We use three weather attributes (ambient temperature, GHI, and relative humidity) and six similarity measures described in Section 2.1. We assign equal weights to the attributes and similarity measures.

The plots of ambient temperature for the top two most similar day pairs which are a paired dates of May 25th and June 3rd, and a paired dates of May 26th and June 3rd as shown in 2(a) and 2(b). The plot for date pair with the lowest similarity score, May 9th and June 4th, is shown in Figure 2(c). The plots for the most similar days show that the time series follow similar patterns and have minimal distance between each other, while the plots for dissimilar days show one time series with a distinct pattern than another and the distance between each other is rather large. For instance, the pair date of May 25th and June 3rd has very low values of MAE and ED for ambient temperature and relative humidity, but higher values for GHI. This shows promising results in the developed similar weather days computation.
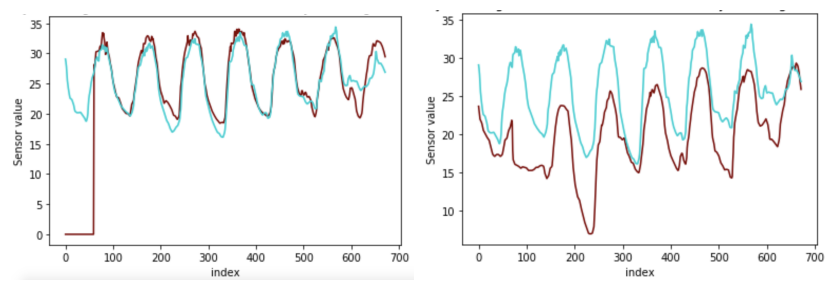

(a) Ambient Temperature May 23th-(b) Ambient Temperature April 25th29th \& May 30th-June 5th May 1st \& May 30th-June 5th

Figure 3: Plots of Ambient Temperature shown for each week pair 3(a)-3(b). The first week of every 7 days pair is shown in maroon and the second week in a pair is displayed in blue.

While analyzing the most similar weather days is an effective approach, considering most similar weeks can be also useful. For instance, April 16th and April 21st very similar weather features shown in Figure 2(d). These traits suggest that energy usage patterns would be very similar on the two dates. However the total energy usages on the April 21st is much higher than that of the April 16th. We identify the spike in energy usage on the April 21st is due to the differences in the day of the week. April 16th is a weekday while April 21st is a Easter holiday where participants would likely spend more time at home and use more energy. With these findings, we consider to identify similar weather for weeks.

We found the weeks of May 23rd-May 29th and May 30thJune 5 th to be the most similar in terms of weather patterns which can be seen in Figure 3. Figure 3(a) show the similar patterns of ambient temperature and the similarities are contrasted by the discrepancies occurring the less similar weeks pairs of April 25th-May 1st and May 30th-June 5th. Figure 3(b) shows the low correlations between these two week pairs are evident, which display how different these two weeks are.

\subsection{Simulation Model}

Identifying the pairs of days/weeks with same weather can be challenging, considering the fact that two days are also the same day of the week (e.g. Sunday Vs Sunday). This motivates us to pursue a simulation based model which does not consider similar weather days/weeks. With this model, we estimate the normal energy usage on a single day and 


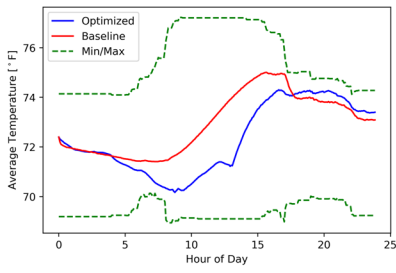

(a) Indoor temperature of HVAC

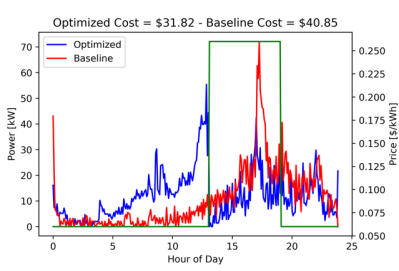

(b) Price of HVAC power
Figure 4: An example graph showing HVAC (a) temperature (baseline, optimized, min and $\max$ ) and (b) price calculated by simulation and optimization model for a single day at every 5 minute interval

during the same day when optimization is not enabled. Our model uses parameters similar to optimization to imitate the operations. After we compute the electrical energy usage for HVAC and $\mathrm{WH}$, we aim to calculate the cost of total energy usage needed based on the current market price of each kilowatt of energy during that particular time period. We find the cost of energy usage by the simulation model after every five minute interval during the day and compare this cost with the cost of energy usage by the HCS optimization shown in Figure 4.

Quantifying cost savings on HVAC on simulation model: We compare the total wattage (in kilowatt-hours) spent by the optimization and simulation for one week given the market price. Figure 5 shows the plots of HCS optimization and simulation model for HVAC in terms of total cost, total energy usage, and peak energy usage per day at neighborhood-level for one week from May 17th to May 23rd when the optimization is enabled.

In total, over the whole week, in comparison to the simulation model, our optimization is able to save $\$ 69.25$ or 433 kilowatt hour at the neighborhood-level. On an average, each of the home saves at least 163 watt-hours of energy and 14 cents in a day.

\section{VISUALIZATION}

The identified similar weather days/weeks pairs enable understanding the impacts of co-optimizing comfort of the home users, energy costs and peak demand which can be visualized through several interactive web-based dashboards.

Quantifying cost savings on HVAC using similar weather pairs: After the computation of similar weather day/week pairs, we select the top 30 pairs that are ranked based on the similarity score. We then extract the power used by the HVAC systems for those similar weather day pair dates. Figure 6 is an interactive dashboard that displays the cost savings due to optimization for each pair of the 30 similar weather pair days at a home and neighborhood-level after applying two different rate structures namely family dwelling
(FD) and TOU rate. This dashboard has 4 widgets that are described below:

(1) HVAC Cost Savings at Neighborhood-level: This widget displays the HVAC cost savings aggregated up to the neighborhood level for each pair of the 30 similar weather pair days.

(2) HVAC Cost Savings at Home-level: This widget shows the HVAC cost savings at a home level but aggregates the costs for all the 30 similar pairs (i.e., around one month savings).

(3) HVAC Total Change: The total change widget sums up the cost savings for the entire neighborhood for all the 30 similar day pairs to get an estimate of the total cost savings for one-month period.

(4) HVAC Overrides aggregated by week: The HVAC overrides widget displays the total number of overrides per home for the weeks of May and June when optimization was on. This widget interacts with the rest of the widgets, so when we exclude homes that decided to override most of the days during the optimization (HCS) enabled weeks, we can instantaneously see the cost saving changes due to the exclusion of those specific homes.

Negative costs within the HVAC total change widget indicate cost savings displayed in red. The cost differences that are positive are displayed in blue which indicate more costs were incurred when optimization was applied. In Figure 6, we see significant cost savings due to HVAC control. We filtered out the homes with the highest number of overrides of the weeks when optimization dispatch is on, which give us more accurate assessment of the cost savings. Note that this approach is not applicable for the WH optimization since the usage of WH is not dependent on the weather.

The similar FD cost comparison for HVAC using similar weather weeks is shown in Figure 7. It is challenging to find similar weather that match every day in a week. Because the weather attributes we use to find similar weather days do not have a close match for all seven days in a week. Thus, the computed HVAC cost savings using similar weather week pairs may not be a fair comparison since HVAC usage is dependent on the weather.

Three-Bucket Dashboard Visualization: Figure 8(a) shows the three-bucket dashboard snapshot for a pair of similar weather days (April 15th and April 21st) at a home-level. The three buckets are described below: Comfort bucket: mainly captures the number of HVAC and WH overrides and number of temperature violations that were encounters within homes. The number of overrides indicates how many times the household members change the settings of HVAC and WH per day and week. For per day, we consider a house has an override on that day and keep the settings for the 


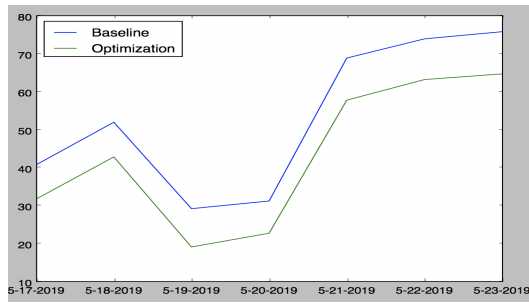

(a) cost

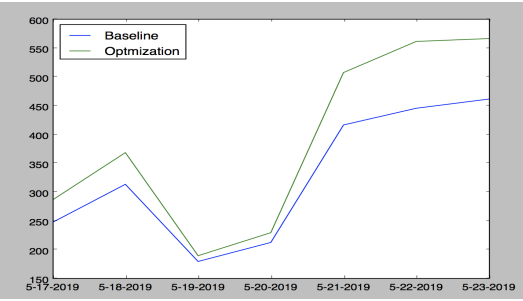

(b) energy in KW

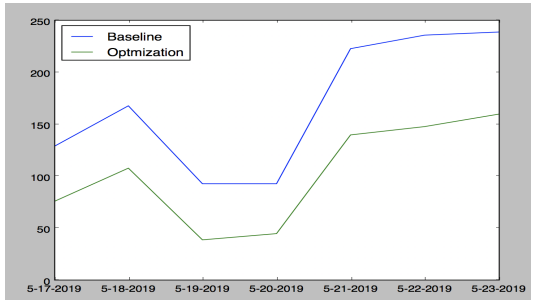

(c) peak energy $(\mathrm{KW})$ in day

Figure 5: Cost, energy comparison of baseline simulation and HCS optimization for one week in May, 2019. Note, although optimization uses more energy than baseline, in terms of cost and peak energy it is lower than the baseline.

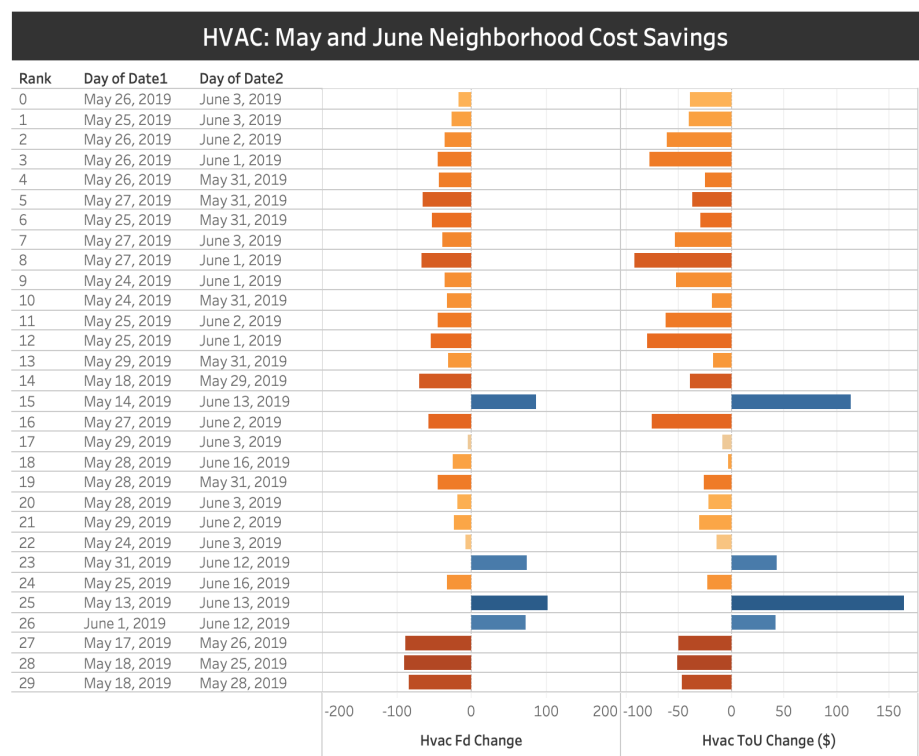

Hvac ToU Change (\$)

$-70.9$

Hvac Fd Change

$-90.5$

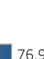

76.9

HVAC: Total Change

Hvac Fd Change (\$)

103.1

Hvac ToU Change (\$)
HVAC : Cost Savings during May and June at Home level

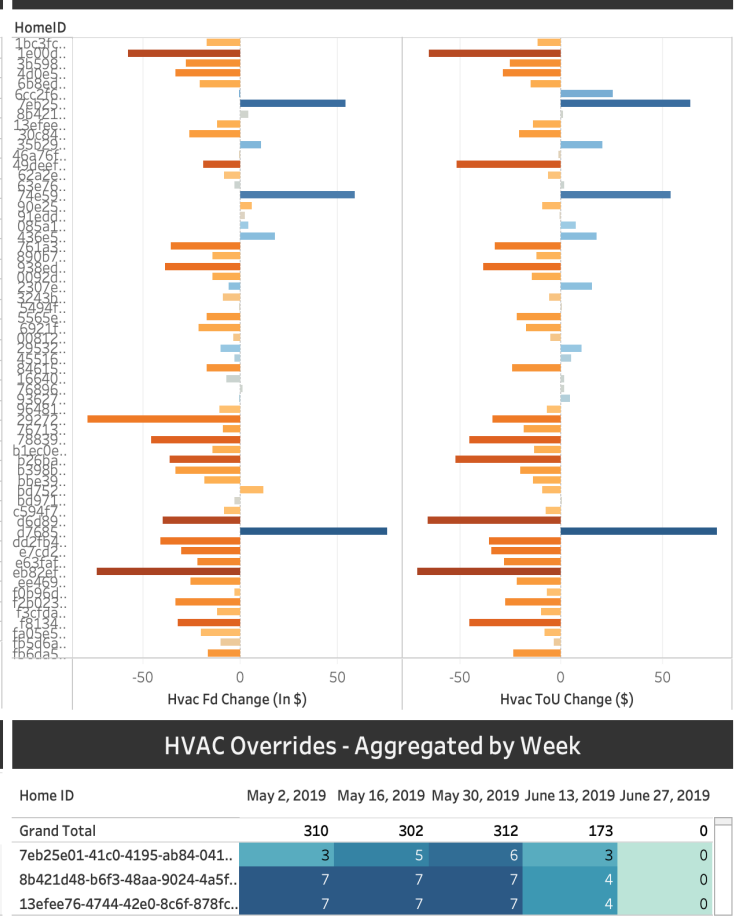

Figure 6: Interactive dashboard to visualize HVAC cost savings at both home and neighborhood-level for the similar weather day pairs.

rest of the day. For per week, we consider how many days of the particular week a house has changed its settings. If we find a house has changed its settings at any time-period of a day in the week, we consider its settings have changed for that day and classify that house as true override for that day of the week. The comfort of the user can be measured by understanding how often the 1) home owner overrides his/her desired comfort range of temperatures within his home during any time of the day referred to as HVAC overrides and 2) home owner overrides the $\mathrm{WH}$ or basically not choose/accept the recommended schedule of operating the
WH appliance referred to as WH overrides. The temperature violations occur when the zonal thermostat temperature is outside the comfort temperature range for each home given the schedule that stores the cooling and heating set points provided by the home owner. To compute the temperature violations, we first extract time series temperature data (every five minutes) for each zone within the home, activity (home, away) and mode (auto, heat, cool) data using the schedule information provided under configuration file. The configuration files contain activity schedule, and cool and heat set points. However, each mode has their own methodology of 


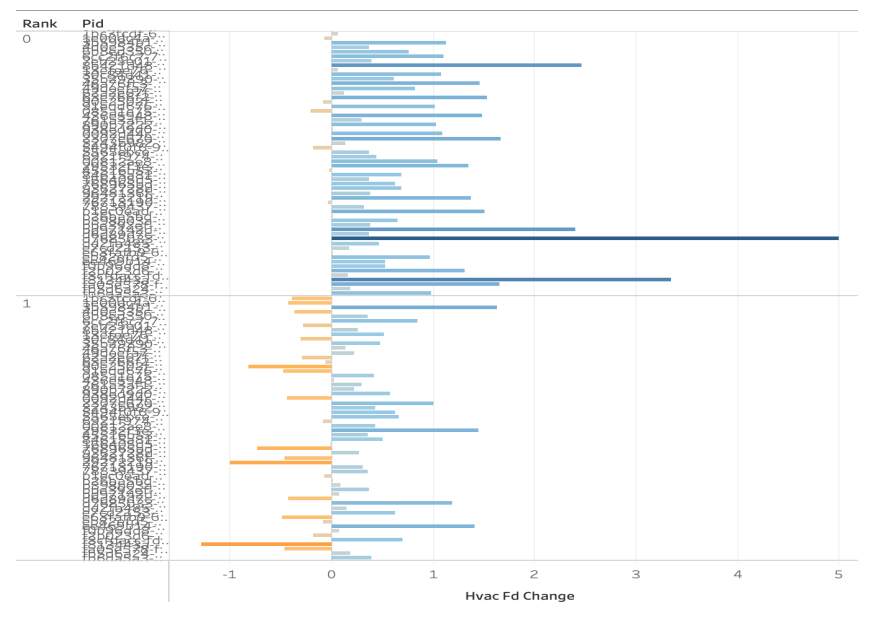

Figure 7: HVAC Cost Difference for Similar Weather weeks

calculate set points. Note that $T_{z}, T_{C S P}, T_{H S P}$ represent the zonal temperature, cooling set point, and heating set point, respectively. The temperature violation is counted when the thermostat temperature falls outside of set points that are specified based on the mode. Each temperature violation can be classified as too warm and too cool.

- Auto mode uses $T_{C S P}$ and $T_{H S P}$ as listed in the configuration file.

- Violation Too Warm: $T_{z}>T_{C S P}$

- Violation Too Cool: $T_{z}<T_{H S P}$

- Cooling mode uses $T_{C S P}$ and $T_{C S P}-2$

- Violation Too Warm: $T_{z}<T_{C S P}$

- Violation Too Cool: $T_{z}<T_{C S P}-2$

- Heating mode uses $T_{H S P}$ and $T_{H S P}+2$

- Violation Too Warm: $T_{z}>T_{H S P}+2$

- Violation Too Cool: $T_{z}<T_{H S P}$

From Figure 9, we notice that the neighborhood has many "too cool" violations in cooling mode during the month of May since the homes will naturally get cooler than the (cooling set point -2 ) and we see very few cooling mode "too warm”.

Energy bucket: This captures the differences between baseline and optimized costs (using the FD \& TOU rate signals), and the difference between baseline and optimized energy usage and forecasted power usage accuracy. FD rate plan is typically for the standard residential customers which has a monthly base charge and a rate that changes based on the monthly electricity usage and the time of the year. The negative difference shown in the table for energy usage or energy cost means that there are energy or energy cost saving. The plots of actual and forecast power usage for HVAC and $\mathrm{WH}$ given the pair dates with the price signal are displayed in this bucket.

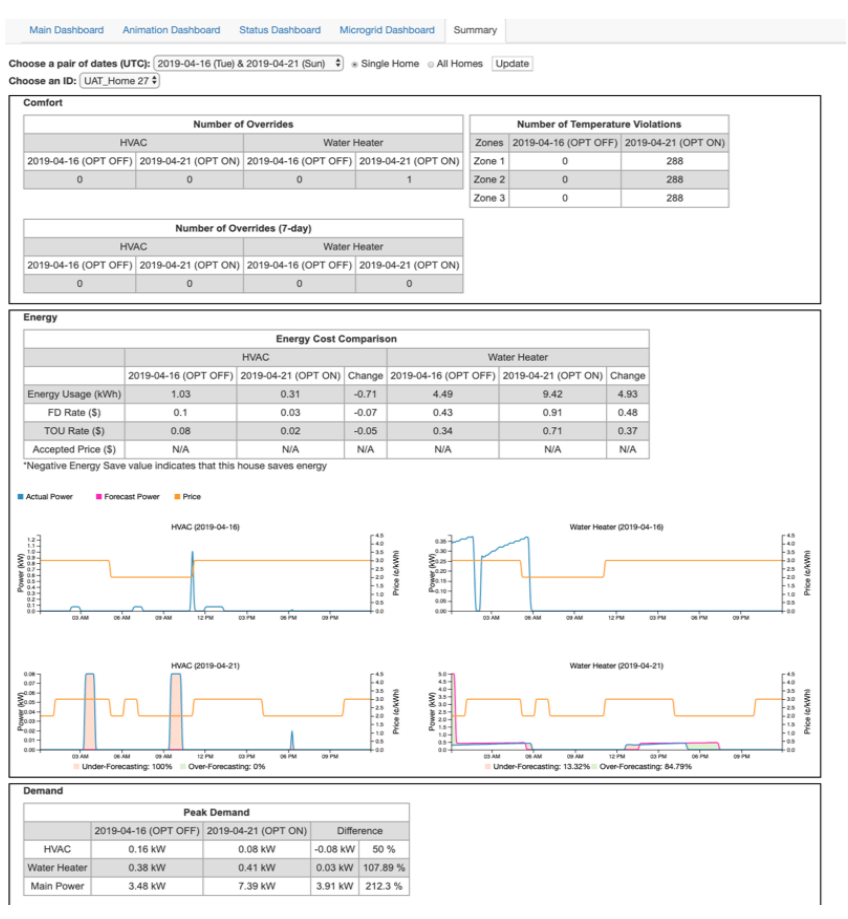

(a) three-bucket dashboard

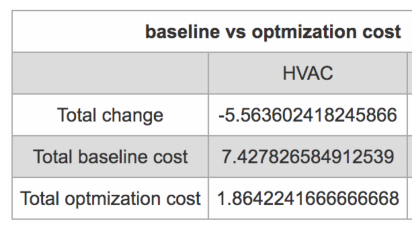

(b) cost saving table of HVAC

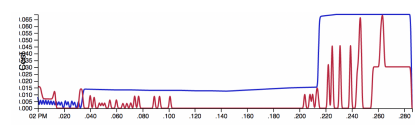

(d) energy cost on HVAC in a day

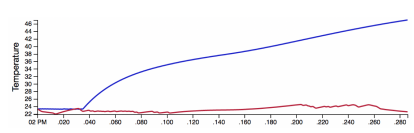

(c) HVAC temperature
Figure 8: (a) displays an interactive dashboard for three-bucket visualization using similar weather or statistical model. (b)-(e) displays an interactive dashboard for three-bucket visualization using simulation model.

Demand bucket: This focuses on the collected channel data for the peak demand of HVAC, WH, and main power of given dates. The difference of peak demand for each component is calculated and converted into percentage. Figure 10 shows the HVAC peak power analysis given two pairs of similar days (May 17th and May 26th, and May 28th and May 25th). The dates that optimization enabled have lower HVAC power demand peak compared to the dates with no optimization applied when the peak price signal is shown. 


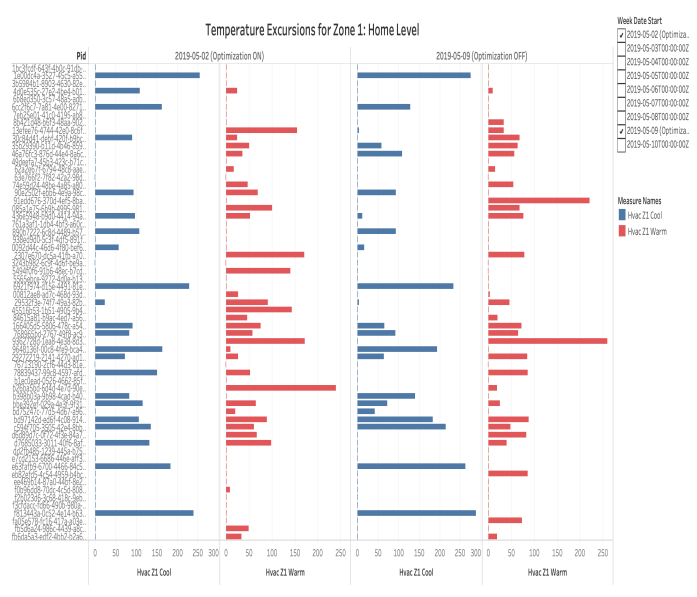

Figure 9: Comparison of temperature excursions between two days of May when the optimization is enabled and disabled respectively at home-level. Red indicates "too warm" and blue indicates "too cool"

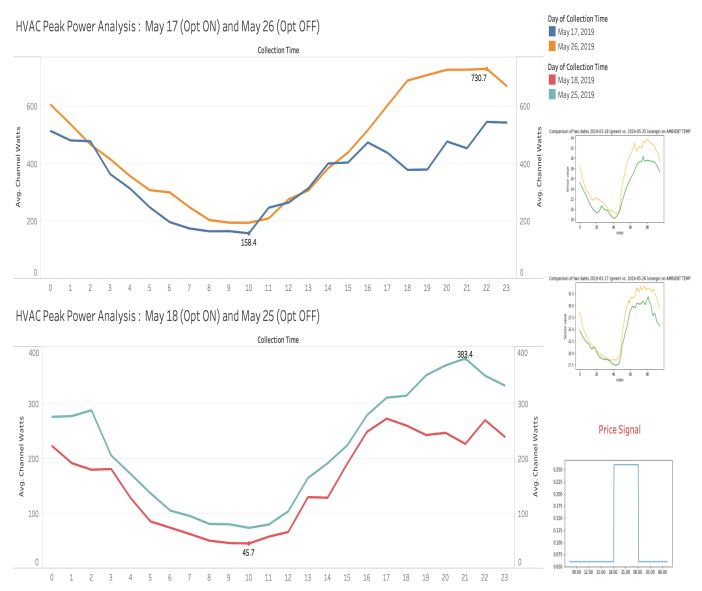

Figure 10: HVAC Peak Power Analysis in May

Dashboard for simulation model: In Figures 8(b)- 8(e), we visualize the price graph and power graph in a three-bucket modeling dashboard to compare the performance of HCS with the simulation model.

\section{DISCUSSION}

While the similar weather day/week pairs identification method has provided optimistic results, there are some challenges that will need to be addressed in the future. As an example, one of the drawback with the similar weather week identification method is that the weather similarity curves between two weeks may not be as close as the curves between two similar days. Our results indicate that two closest days that matched up are far more similar to one another than the 2 weeks that matched mainly due to the fact that the probability for weather patterns to be the same for a day is much greater than being the same for a week period. Additionally, identifying two similar weather days is less challenging than identifying two similar weeks since the pool of dates to compare is seven times greater than the pool of weeks.

Also, the paired dates are computed using weather data (temperature, humidity etc.) that is recorded every 5 minutes which is the finest resolution data that we currently store within the database. If we are identifying similar pair days, we have many pairs for which we can compute weather similarity (by picking one day from the dispatch week and one day from the non-dispatch week). If we are identifying similar pair weeks, then we have lesser pairs to compute but the time-series weather data used for the week is seven times more than a day. So, the amount of data we are using for the computation remains the same, although computing similar pair days can be easily parallelized than similar weeks computation algorithm.

We also noticed that the participant's energy usage pattern on different days of the week could bias the results. For example, if two days had very similar weather patterns with HCS enabled and disabled, but occurred on different days of the week, the actions of the participants would skew the cost associated with optimizing climate conditions within the home. A participant's energy usage on weekends and weekdays could be significantly different, even though the two days had similar weather patterns.

However, using a week long of energy usage data may better capture the usage pattern than a day data. Due to these constraints posed by similar weather days/weeks detection methods, a combination of approaches (including simulation based) is recommended for quantifying cost savings due to optimization. Overall, using both methods of analysis allowed us to have a better picture of the results of optimization.

\section{CONCLUSION}

Rapid grid modernization along with growth of IoT, Big Data and artificial intelligence technologies are enabling electric utilities to perform automatic scheduling and control of smart appliances such as HVAC and WH systems which accounts for a majority of residential load within smart neighborhoods to minimize energy costs. Quantifying economic savings requires accurate baseline data (without optimization applied) to effectively compare with the energy costs due to optimization and control. In this paper, we proposed a novel method of identifying similar weather day/week pairs to perform a fair comparison of energy costs with and 
without optimization applied at home and neighborhoodlevel within high-performance residential homes. With the identified two similar weather days in May and June, the energy cost savings with optimization applied to HVAC at neighborhood-level is approximately $\$ 700$ within a month period. We filtered homes that overrides HVAC settings most of the days and found that the cost savings is still significant. We also demonstrated a simulation based method to quantify cost savings and showcased our results through interactive visualization dashboards.

For future work, since the simulation model based approach of quantifying cost savings does not depend on weather, we plan to use this model to quantify cost savings due to WH (since WH usage is not dependent on weather) as well as a part of future work. Future studies will also focus on a promising deep learning based approach for quantification of cost savings through optimized control of HVAC and WH appliances based on electricity price signal. A long-term short-term (LSTM) network based analysis will be used to predict the consumption pattern of the household power using historic data during normal operation without optimization. Due to high-frequency datasets available, these predictions will be fairly accurate and will be compared against the actual power consumption of the neighborhood to provide reasonable estimates of utility-cost reductions obtained due to optimized control of smart appliances.

\section{REFERENCES}

[1] Hervé Abdi. 2007. The Kendall rank correlation coefficient. Encyclopedia of Measurement and Statistics. Sage, Thousand Oaks, CA (2007), 508-510.

[2] Jacob Benesty, Jingdong Chen, Yiteng Huang, and Israel Cohen. 2009. Pearson correlation coefficient. In Noise reduction in speech processing. Springer, 1-4.

[3] Chrissy Callahan. 2019. How to save money on your electricity bill using smart home appliances. https://www.today.com/home/best-smarthome-systems-save-money-electricity-t127011.

[4] Southern Company. [n. d.]. Smart Neighborhood $^{\mathrm{TM}}$. https://www.southerncompany.com/ campaign/imagine/imagine-smart-energy-for-betterliving.html.

[5] EIA. [n. d.]. EIA's residential energy survey now includes estimates for more than 20 new end uses. https: //www.eia.gov/todayinenergy/detail.php?id=36412

[6] Melike Erol-Kantarci and Hussein T Mouftah. 2010. TOU-aware energy management and wireless sensor networks for reducing peak load in smart grids. In 2010 IEEE 72nd Vehicular Technology Conference-Fall. IEEE, $1-5$.
[7] Thomas D Gauthier. 2001. Detecting trends using Spearman's rank correlation coefficient. Environmental forensics 2, 4 (2001), 359-362.

[8] Luis Hernández, Carlos Baladrón, Javier M Aguiar, Lorena Calavia, Belén Carro, Antonio SánchezEsguevillas, Diane J Cook, David Chinarro, and Jorge Gómez. 2012. A study of the relationship between weather variables and electric power demand inside a smart grid/smart world framework. Sensors 12, 9 (2012), 11571-11591.

[9] Eamonn Keogh and Chotirat Ann Ratanamahatana. 2005. Exact indexing of dynamic time warping. Knowledge and information systems 7, 3 (2005), 358-386.

[10] Teja Kuruganti. 2017. Connected Neighborhood. https: //tinyurl.com/y4k2qwfm.

[11] Xiao-Li Meng, Robert Rosenthal, and Donald B Rubin. 1992. Comparing correlated correlation coefficients. Psychological bulletin 111, 1 (1992), 172.

[12] Jordi Serra, David Pubill, Angelos Antonopoulos, and Christos Verikoukis. 2014. Smart HVAC control in IoT: Energy consumption minimization with user comfort constraints. The Scientific World Journal 2014 (2014).

[13] Iain Staffell and Stefan Pfenninger. 2018. The increasing impact of weather on electricity supply and demand. Energy 145 (2018), 65-78.

[14] Michael Starke, Helia Zandi, Teja Kuruganti, Jeffrey Munk, Heather Buckberry, Jim Leverette, and Joni Hall. 2019. Agent-Based System for Transactive Control of Smart Residential Neighborhoods. In 2019 IEEE PES Transactive Energy Systems Conference Proceedings.

[15] Israr Ullah and DoHyeun Kim. 2017. An improved optimization function for maximizing user comfort with minimum energy consumption in smart homes. Energies 10, 11 (2017), 1818.

[16] Cort J Willmott and Kenji Matsuura. 2005. Advantages of the mean absolute error (MAE) over the root mean square error (RMSE) in assessing average model performance. Climate research 30, 1 (2005), 79-82. 